\title{
Severe systemic scleroderma with multiple organ involvement in a 45 -years-old patient
}

\author{
Violeta Diana Oprea', Violeta Claudia Bojinca', Grigore Balosin'2, Ruxandra Nicoleta Ciofu³, \\ Ruxandra lonescu' ${ }^{1}$ \\ ${ }^{1}$ Department of Internal Medicine and Rheumatology "Sf. Maria” Hospital, Bucharest, Romania \\ 2 Department of Cardiology, "Sf. Maria" Hospital, Bucharest, Romania \\ ${ }^{3}$ Department of Plastic Surgery, "Sf. Maria" Hospital, Bucharest, Romania
}

\begin{abstract}
Systemic scleroderma has inflammation and fibrosis playing a crucial role and eventually leading to severe functional failure and damage of multiple organs. Three primary mechanisms are mainly involved in the pathogenesis of scleroderma: vascular anomalies, excess fibrosis, and autoimmune phenomenon.

The present case study shows a severe cardiac dysfunction with low cardiac output syndrome (LCOS) with cardiac, hepatic and renal impairment, peripheral ischemia, neurologic symptoms associated or even aggravated by a probable severe toxicity to azathioprine therapy. Currently there is no cure for systemic sclerosis, the aims of treatment are- as recommended also by EULAR: to relieve symptoms, to prevent the condition from progressing, as much as possible, to detect and treat complications early and to minimize any disability.
\end{abstract}

Keywords: systemic sclerosis, cardiac failure, drug hepatotoxicity

\section{INTRODUCTION}

Being named from Greek words "sclero" ("hard") and "dermis" ("skin"), scleroderma is a connective tissue disorder with 2 primary types: a localized one, where skin is mainly affected- but also bones and muscles may be involved, and the systemic sclerosis, which can affect multiple organs and systems: heart, digestive tract, respiratory system, kidneys. The evolution, the severity, the response to therapy and the prognosis can vary from patient to patient $(1,2)$.

Systemic scleroderma affects women more (female: male ratio estimated at 4:1-10:1, depending on age and ethnicity) and recently the pathogenic mechanisms were moving the focus from the mainly fibrotic pathway to the central role of endothelial damage together with participation of immune response cells and mediators, fibroblasts, components of the extracellular matrix. There have been described 2 clinical subsets of the systemic scleroderma: the diffuse cutaneous form and the limited cutaneous one. Most pathogenesis studies emphasized on a complex role of genetics (OX40L gene polymorphism correlates with systemic scleroderma) and external environmental factors (like some organic solvents, silica) in the abnormal activation of the immune system causing tissue injury and blood vessel impairment determining accumulation of excess collagen in the intercellular space (3-5).

During the course of the disease patients may develop vascular complications, inflammatory myopathy, cardio and pulmonary damage, articular involvement, digestive involvement. Fatalities occur especially in cases of serious cardiovascular or pulmonary disease, making systemic scleroderma the single connective tissue disorder with the worst survival prognosis - a 3-year survival rate of $47-56 \%$ $(6,7)$.

\section{CASE PRESENTATION}

The present report is introducing the case of a 45 years old female patient diagnosed with systemic sclerosis at the age of 41 when she already had cutaneous, 

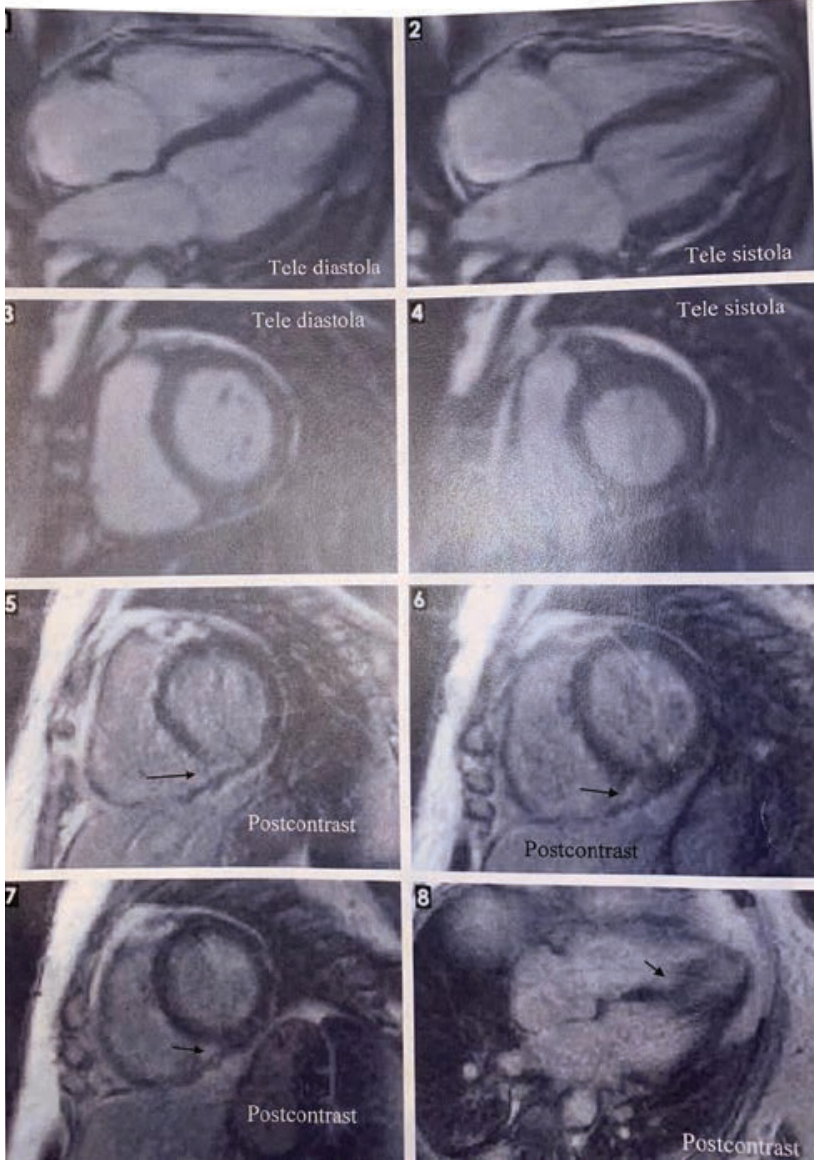

FIGURE 1. Cardiac IRM evaluation 3 years before current presentation - myocardial fibrosis of the left ventricle already present

pulmonary, cardiac, renal and articular involvement. The patient presented at the Emergency Room at the "Sf. Maria" Hospital in Bucharest in September 2020 after multiple medical consultations (hospital and outpatient clinics) during the difficult months of COVID-19 pandemics when chronic patients had limited access to their treating medical teams.

From the personal pathological history it is noticeable that in 2017 a coronarography revealed no significant lesions, in 2018 a cardiac IRM showed myocardial fibrosis of the left ventricle and an EF of $50 \%$ (figure 1), and in 2018 an acute renal failure enforced renal dialysis procedure in the context of systemic scleroderma affecting the kidneys.

The patient underwent pulses of cyclophosphamide in the year prior to hospital admission in our clinic. Recent health issues related to the background disease, 2-3 months before presenting to our Emergency Room, included a severe progressing dyspeptic syndrome with nausea, loss of appetite, vomiting, also asthenia and fatigability and breathing difficulties.

For scleroderma and the associated morbidities patient's therapy was, before the onset of these symp- toms, azathioprine $50 \mathrm{mg} 1 \mathrm{tb}$ daily, prednisone $5 \mathrm{mg}$ daily, furosemide $40 \mathrm{mg}$ daily, nebivolol $5 \mathrm{mg}$ daily, and iron supplements. The special pandemic COVID-19 situation limited all patients' access to their treating physician, a critical issue for all chronic patients but especially for those suffering from special disorders (as is scleroderma, which often requires a more specialized doctor and also multiple consultation from a multidisciplinary medical team); this affected our patient's medical surveillance and direct monitoring of her condition, so she increased her azathioprine daily dose first to 2 X $50 \mathrm{mg}$ and then, due to worsening of her symptoms, to 3 X $50 \mathrm{mg}$. In the meantime, she presented to the ER department in another hospital complaining about dyspnea at minimal efforts, fatigability, acro-paresthesia in both her arms and feet, constant nausea and several episodes of vomiting daily, an intermittent tremor at the level of upper limbs, general weakness which seriously impacted the quality of life. Lab tests showed she developed again renal failure, also anemia and biologic inflammatory syndrome. The therapeutic approach consisted of medication to correct these, so corticoid therapy pulse was recommended associated with anti-anemic medication. Also further investigations with relevant data included:

$>$ upper digestive endoscopy diagnosing Los Angeles grade B esophagitis, gastric stasis; gastroenterologist attributed the clinical symptomatology to either a digestive manifestation of scleroderma or a secondary adverse event following the intolerance of the iron corrective supplement that was administered

cardiologic evaluation:

o echocardiography indicated multiple damages at both left and right sides: left ventricle failure class II NYHA, moderate left ventricular systolic dysfunction, cardiac fibrosis, improbable pulmonary hypertension

o EKG showing sinus rhythm with major right fascicular block, left anterior hemiblock, sequelae of acute inferior myocardial infarction.

A second emergency hospital admission in a different clinic (10 days after the previous one) with severe symptoms suggestive for an acute abdomen includes an abdominal ultrasound (diffuse hepatic steatosis, gallbladder with $6 \mathrm{~mm}$ double thick walls, moderate biliary residue / no gallstones, free intraperitoneal $30 \mathrm{~mm}$ fluid present in the lower abdomen) and a surgical evaluation which does not rec- 
ommend any invasive cure, so the patient started hydro-electrolytic rebalancing therapy, antiemetic medication, proton-pump inhibitors, antalgic drugs. The patient decides for on-request discharge against medical advice so the previous scleroderma therapeutic scheme is continued at home, with limited efficacy and aggravation of the symptoms.

Upon extreme worsening of the vomiting and the severe alteration of the clinical status, with dyspnea and dry cough, the patient presents to the Emergency Room at "Sf. Maria" Hospital, where the admission is decided in the Medical Department.

Clinical exam:

- altered general status, characteristic facies of "byzantine icon" including thin lips, small mouth, thin nose and perioral wrinkles, skin thickening with puffy fingers in both upper and lower limbs, sclerodactyly, digital ischemia, Raynaud phenomenon with acrocianosys, cracking when knee joints are mobilized;

- normal pulmonary auscultation with physiological vesicular murmur and no bronchial rales added; $\mathrm{Sp} \mathrm{O} 2=98 \%$;

- $\mathrm{BP}=120 / 90 \mathrm{~mm} \mathrm{Hg}, \mathrm{AV}=80 \mathrm{bpm}$ in sinus rhythm but with numerous supraventricular extrasystoles, systolic murmur

The patients continued to present nausea with episodes of incoercible vomiting, heartburns and pain located in the right hypochondrium, which, coroborated with lab tests showing iron deficiency anemia, hepatocytolisis, altered renal lab results, raised the suspicion of acute abdomen.

Abdomino-pelvic CT examination diagnosis: CT appearance suggestive for an acute inflammatory substrate at the gallbladder level - to be correlated with clinical-biological data and with surgical evaluation, small amount of fluid in the pelvis, multiple areas with bilateral postero-basal "tree in bud" appearance, with small "matte glass" areas associated with the left side, with non-specific CT aspect - to be further monitored by imaging.

Surgical evaluation and monitoring in 2 consecutive days concluded that the acute alithiasic cholecystitis without any sign of peritoneal irritation in the context of multiple organ dysfunctions should be managed by medical approach (antibiotics, hydro-electrolytic rebalancing therapy, antiemetic medication, local ice applications) and does not require an emergency surgical intervention.

In the first 48-72 hours after hospital admittance the patient's medical condition is worsening, she presented:

$>$ Aggravation of symptoms: dyspnea, acrocyanosis, nausea with incoercible vomiting, precordial pain and palpitations

Aggravating impairment of her cardiac function, objectivized by echocardiogram $(\mathrm{LVEF}=$

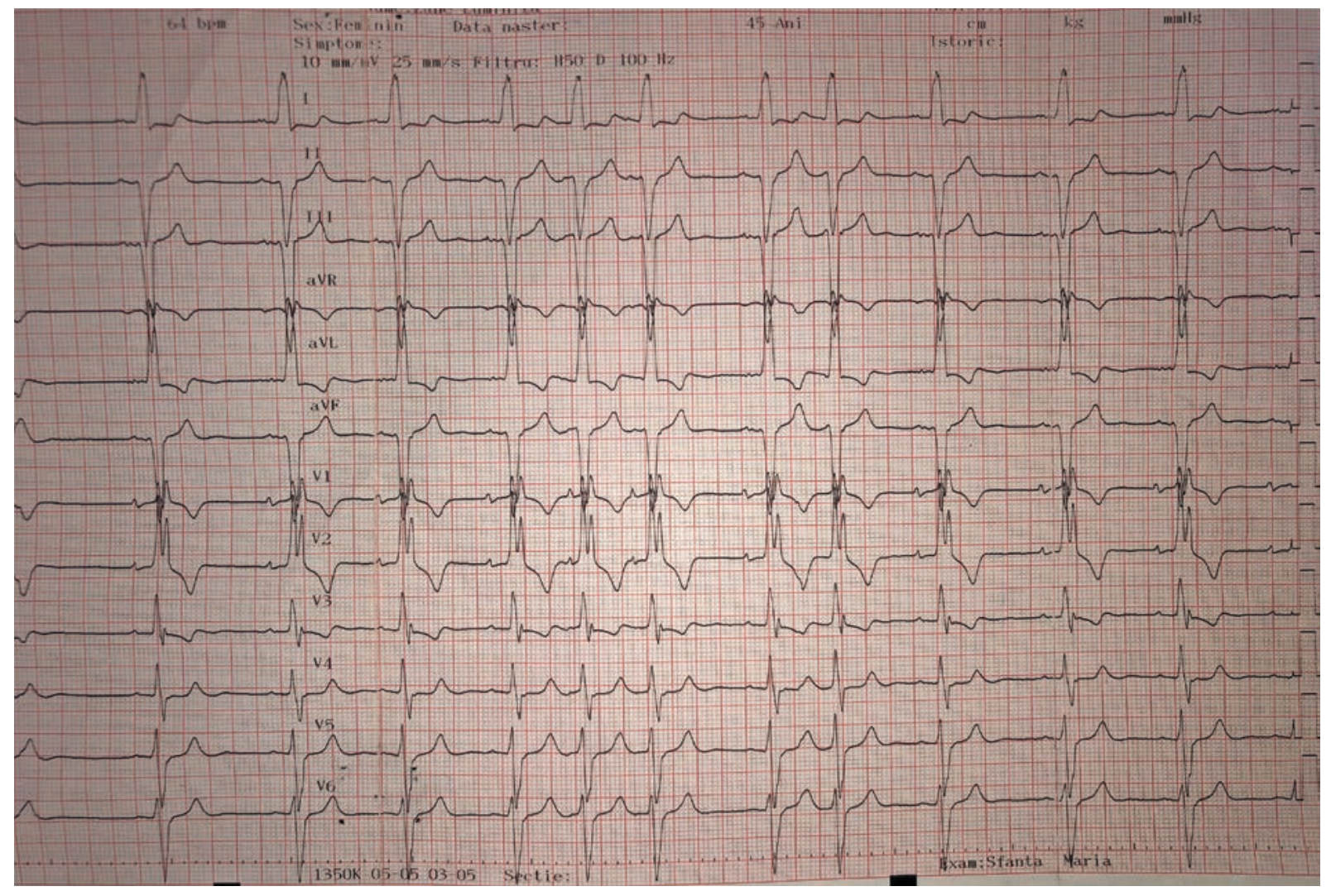

FIGURE 2. ECG at current stage of the disease, showing significant cardiac injury 


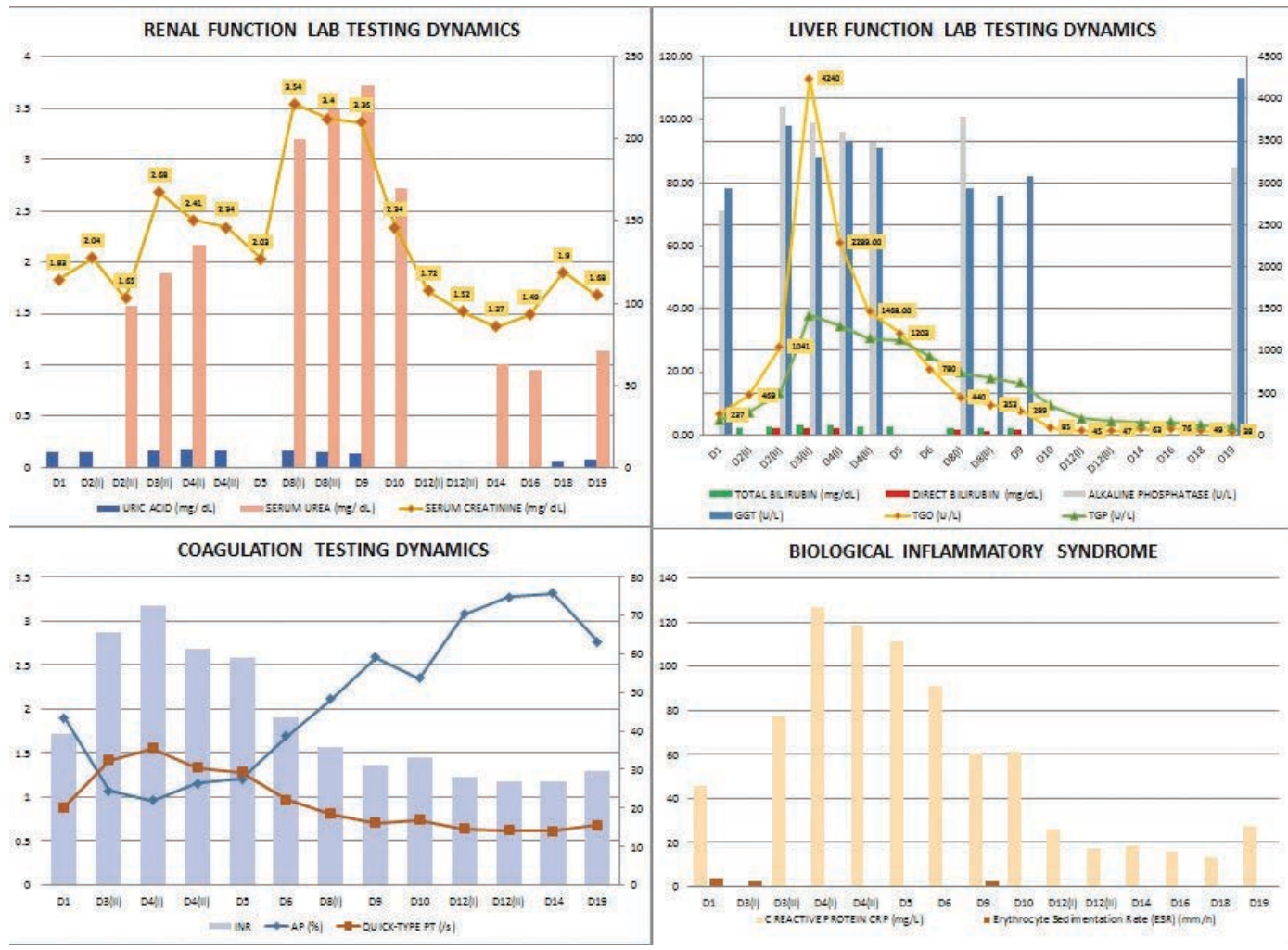

FIGURE 3. Relevant biology evolution during 2020 hospitalization

$15 \%$, Left ventricular outflow tract velocity time integral-LVOT VTI $=7-8 \mathrm{~cm}$, mitral regurgitation grade II and severe tricuspid regurgitation) and a NT-pro BNP test $=10,557.2 \mathrm{pg} /$ $\mathrm{ml}(\sim 85$ X ULN)

EKG monitoring showed multiple supraventricular extrasystole flaps, bifascicular block right bundle branch with anterior fascicle of the left bundle branch - and left ventricular hypertrophy (figure 2).

$>$ A severe hepatocytolisis (an increase in TGO up to $4,240 \mathrm{U} / 1$ - representing $>120 \mathrm{ULN}$ and in TGP to $1420 \mathrm{U} / 1->40 \mathrm{ULN}$ ) (figure 3).

Renal dysfunction (level of serum creatinine increased up to $3.54 \mathrm{mg} / \mathrm{dl}$ )

\section{$>$ Hydroelectrolytic imbalance}

$>$ Biologic inflammatory syndrome with $\mathrm{C}$ reactive protein (CRP) up to $127.15 \mathrm{mg} / \mathrm{l}$ and procalcitonin in the interval $0.5-2.0 \mathrm{ng} / \mathrm{ml}$

The diagnosis completed as: probable severe toxicity to azathioprine therapy associated with Low cardiac output syndrome (LCOS) with cardiac, hepatic and renal impairment, peripheral ischemia, neurologic symptoms (drowsiness and somnolence, fatigue).

Cardiac failure was a severe clinical manifestation being caused by myocardial involvement- common in systemic sclerosis, which is considered to be related mainly to abnormal vasoreactivity and microvascular injuries such as transient coronary artery spasm leading to repeated focal ischemia. The hypothesis of myocarditis in our patient's case is supported by the rapid decompensation of the cardiac parameters (including plasmatic biomarkers) and also by preexisting myocardial fibrotic injuries revealed by ECG and MRI. In systemic sclerosis with cardiac involvement, hypoperfusion is commonly present due to vasospasm but also to concentric intimal hypertrophy of small coronary arteries, thus cardiac failure is self-sustaining and requires strong inotropic support. A serious challenge was represented by nausea and uncontrollable vomiting which prevented the oral administration of diuretics.

The hepatocytolitic syndrome could be considered of complex etiology: toxic (due to azathioprine therapy) but also ischemic- related (the complex vascular system of the liver and its high metabolic activity render it vulnerable to circulation disturbances and decreased oxygen saturation).

Therapeutic approach included O2-therapy, diuretics and carvedilol, inotropic support with dobutamine, anticoagulant medication, hydro-electrolytic rebalance, antibiotics. After 8-10 days the medical condition and vital signs improved but the peripheral hypoperfusion became the main complaint of the patient so, once the liver and renal function improved, 
prostaglandin vasodilator is initiated (alprostadil). Upon complex therapy aiming at improving the cardiac function, some adjustments in the medication scheme was necessary in order to cope with hypocoagulation effects (hematuria, epistaxis) or peripheral spasm resulting in digital necrosis areas.

Another echogardiogram performed after 20 days of hospitalization revealed the moderate improvement in the cardiac function: $\mathrm{EF}=24 \%$, LVOT VTI $>9 \mathrm{~cm}$, slight improvement in the valvular efficiency (figure 4). Also on ECG a decrease in the number of supraventricular extrasystoles was noticed $(<4-5 /$ $\min )$.

The patient is continuing the vasodilator therapy to limit as much as possible the trophic disordersdigital ulcerations evolving in 3 of patient's toes (figure 5).

During the alprostadil therapy (after 11 days of intravenous infusions) patient is presenting again cardiac symptoms- reports of palpitations associated with an arrhythmic episode of paroxysmal atrial tachycardia with 2:1 atrioventricular block that needed conversion with iv beta-blockers.

Upon clinical improvement the patient was discharged with treatment recommendation for mycophenolate mofetil, diuretics, conversion enzyme in-

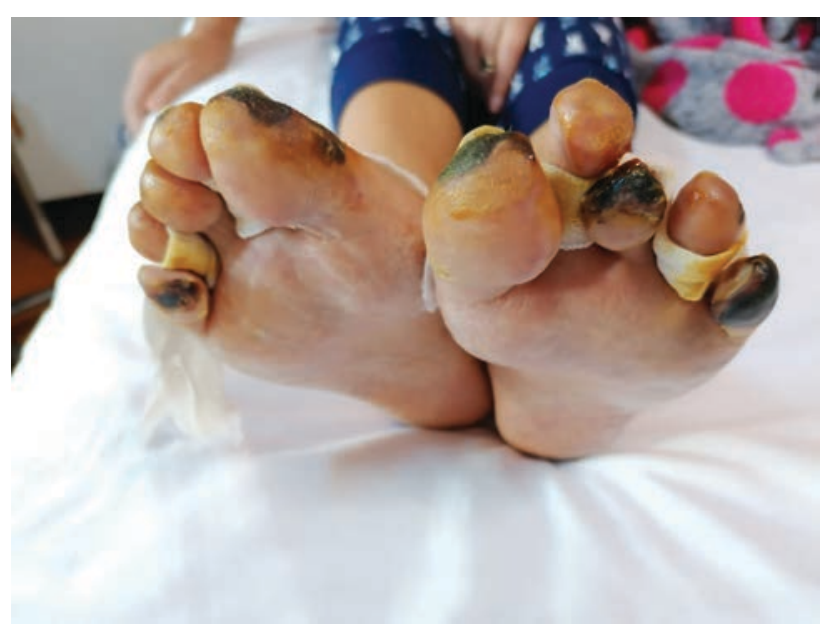

FIGURE 5. Digital ulcerations of the toes before vasodilator therapy

hibitors, anthiarrytmics, iron supplements, proton -pump inhibitors, periferal vasodilators. She was the re-admitted to resume alprostadil infusion for another 20 days and to be treated by the plastic surgeon for the toe ischemia, which evolved to ulcers for 4 toes, bilaterally.

Further in the disease evolution, the patient presents after another month to our clinic with nausea\& vomiting, asthenia, cough, a lipothymic episode causing a frontal cerebro-cranial traumatism. After being diagnosed with a mild form of COVID-19 (by RT-

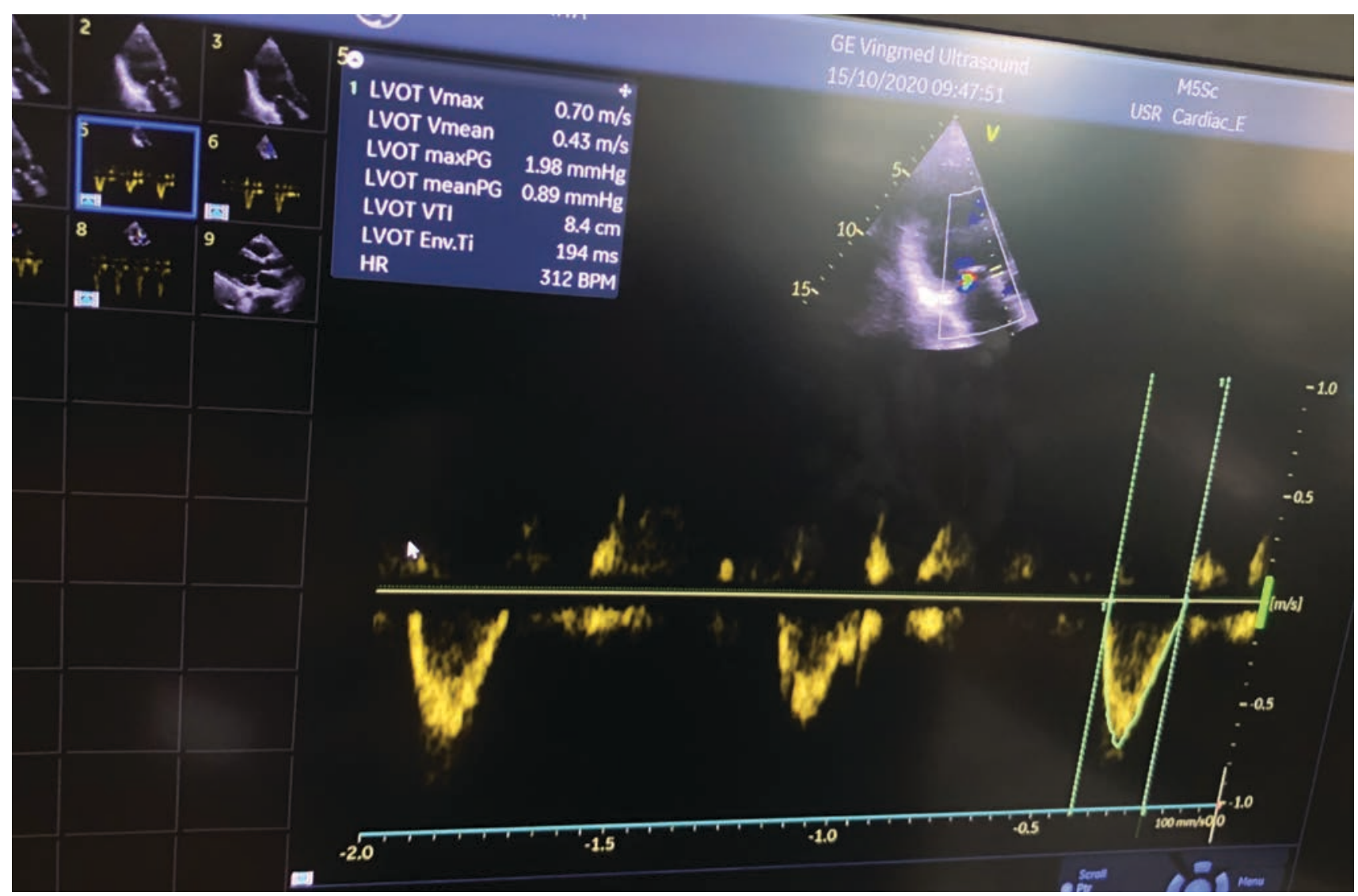

FIGURE 4. Echocardiography in 2020 after 20 days of hospital therapy 
PCR positive SARS-CoV-2 testing), she is transferred to a COVID-support hospital where a thoracic $\mathrm{CT}$ reveals discrete interstitial pulmonary infiltrates located diffusely and peripherally affecting $<10 \%$ of bilateral parenchyma. The patient was treated for 8 days based on current local COVID-19 protocols; antitussive treatment, antiinflammatory medication, supportive vitamins were added to preexisting therapy for background disease (micofenolat mofetil, corticotherapy, diuretics, pentoxifylline etc.). Under therapy the coronavirus-related clinical status improved - Sp O2 evolved from $96 \%$ at hospital admission to $99 \%$ upon discharge, cough ameliorated, asthenia diminished.

Currently the patient is continuing alprostadil infusion course, presenting a well- improved clinical status. Her cardiac evaluation revealed the severe yet stabilized impairment of the myocardia: a left ventricle mildly dilated with normal wall thickness, severe global hypokinesis of LV and an EF between 25 and $30 \%$, pseudonormal LV filling pattern, LVOT VTI $=10.3 \mathrm{~cm}$. Peripheral hemoperfusion responded very well to specific therapy (figure 6).

\section{DISCUSSIONS}

The natural evolution of scleroderma is different from patient to patient, but the morbidity and mortality statistics correlate with the type of the disease:

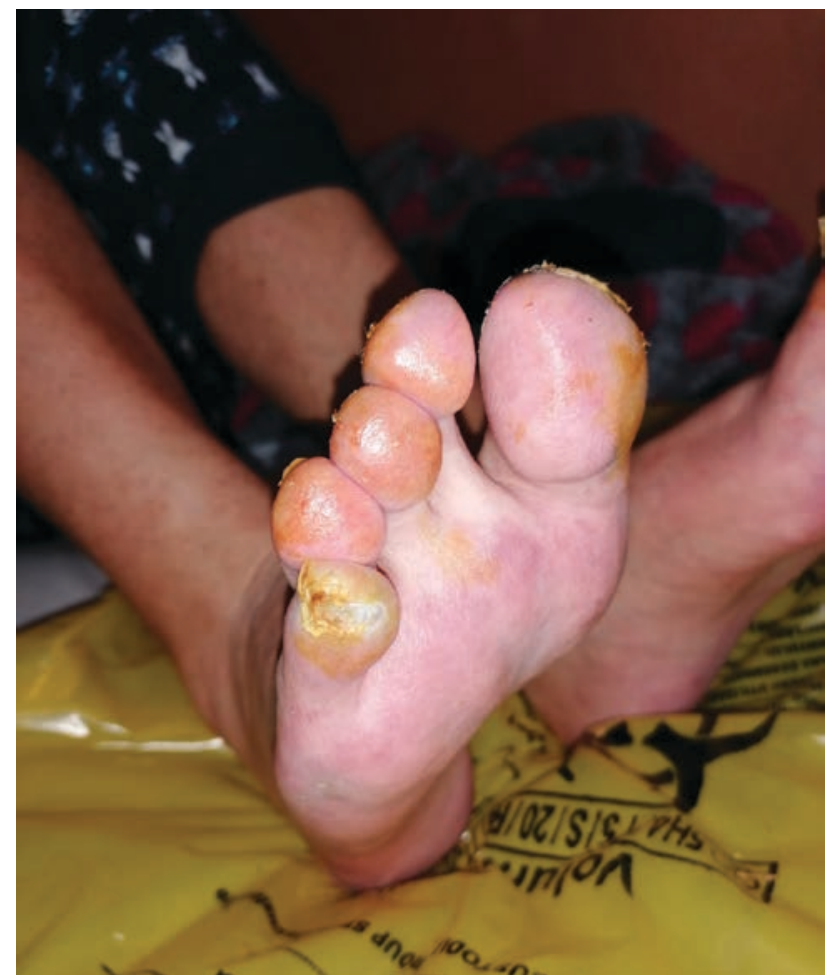

FIGURE 6. Improvement in peripheral perfusion (toe ulcerations) after 2 months of vasodilator treatment patients with cutaneous disease live longer, 5-year survival being estimated to $\sim 80 \%$ for diffuse cutaneous disease and $\sim 90 \%$ for limited cutaneous form. In systemic sclerosis the main characteristics are: microvascular damage, fibrosis of multiple organs, dysregulation of both innate and adaptative immunity. The causes of systemic scleroderma-related deaths evolved over the last decades (as monitoring, surveillance and targets of therapy evolved), with cardiac and respiratory complications currently being considered the leading causes of death (8). The results of a 2019 meta-analysis (9) correlated with the outcomes from a French cohort study of prognosis factors associated with a worse prognosis elements like age at disease onset, age at diagnosis, male sex, African origin, presence of anti-Scl70 antibodies, kidney involvement, valvular disease, cancer, telangiectasia, anemia, and inflammation. An interesting set of data of this publication (from the perspective of our case study) refers to cardiac involvement and related prognosis described a strong association between survival and valvular disease $(\mathrm{HR}=4.03$ [1.97-8.25], $\mathrm{p}<0.001)$, arrhythmia $(\mathrm{HR}=2.44$ [0.98-6.02], $\mathrm{p}=$ $0.054)$, but also Scleroderma renal crisis $(\mathrm{HR}=3.44$ [2.01-5.89], $\mathrm{p}<0.001$ ) and survival prognosis (9).

The most acknowledged evidence of the participation of the immune system is the presence of different autoantibodies, several of which are present exclusively in this disease and are associated with clinical complications and specific phenotypes (6). Also from our case perspective, early onset of the disease with multiple organ involvement associated with anti SCL 70 antibodies (ELISA, serum) $>200$ U/ $\mathrm{ml}$ and ANA anti-nuclear antibodies (ELISA, serum) $=41.4 \mu \mathrm{g} / \mathrm{ml}$, also a low level of complement $\mathrm{C} 3$ and $\mathrm{C} 4$, suggest a poor prognosis and a difficult course of the disease.

Three primary mechanisms are mainly involved in the pathogenesis of scleroderma: vascular anomalies, excess fibrosis, and autoimmune phenomenon.

Microcirculatory vascular involvement develops as an effect of abnormal interactions between endothelial cells, fibroblasts, and $\mathrm{B}$ and $\mathrm{T}$ lymphocytes. Large amounts of endothelin 1 are secreted by the endothelial cells, resulting in vasoconstriction and fibroblast activation. Furthermore, fibroblasts and activated endothelial cells produce reactive oxygen species that speed up vascular remodeling, leading to the obliteration of small blood vessels. Activated fibroblasts differentiate easily into myofibroblasts, which have increased collagen synthesis ability (10). These result 
into various symptoms and manifestations of scleroderma (11) - not necessarily seen all together in every patient, but most present in the situation of our case:

o skin symptomatology: usually bilateral and symmetrical starting at distal level, with cutaneous thickness, affected nails, inexpressive facies of "byzantine icon", calcinosis, pigmentation disorders and telangiectasia;

o Raynaud's phenomenon- arterial vasospasm after exposure to cold/ temperature changes, resulting in syncopal pallor with anesthesia, cyanosis paresthesia, and late erythematous, painful phase with digital ulcerations;

o gastrointestinal involvement: Gastroesophageal reflux disease (GERD), gastroparesis, with concomitant nutritional deficiencies (folate and vitamin B12), malabsorption (steatorrhea), and pseudo-obstruction;

o cardiac manifestations including damage of the coronary microcirculation, myocardial disease, conduction system defects, arrhythmias, pericardial disease;

o pulmonary impairment complications like interstitial lung disease (imagistic findings: the ground-glass/ appear linear or reticular images, septal or intralobular/ honeycomb appearance with traction bronchiectasis), pulmonary arterial hypertension (occurs in up to $13 \%$ of patients with systemic scleroderma);

o renal impairment: hypertension, oligo/anuric acute renal failure

o musculoskeletal manifestations at the level of joints, tendons, muscles (myalgia, weakness, more rarely myositis)

A particular situation of our case study was the overlap of aggravation of scleroderma complications with toxicity of azathioprine that was dose-related. Among the known side effects of the therapy are hepatic impairment (various pathologies including hepatocytolisis, cholestasis, destructive cholangitis, peliosis hepatitis, perisinusoidal fibrosis, and nodular regenerative hyperplasia), gastrointestinal disorders (e.g. nausea, anorexia, vomiting), blood and lymphatic system disorders (leucopenia, thrombocytopenia, anemia, pancytopenia and aplastic anaemia, megaloblastic anaemia, erythro hypoplasia, agranulocytosis), infections and infestations, neoplasms benign and malignant (including cysts and polyps) (12).

Groups of experts have tried to come to a consensus regarding treatment for specific organ involvement in scleroderma. EULAR's recommendations for the treatment of systemic sclerosis aim to guide treatment for patients based on evidence and clinical experience from worldwide experts. The modern medical management of the disease is still a quest for more effective approaches. Currently there is no cure for systemic sclerosis, the aims of treatment are: to relieve symptoms, to prevent the condition from progressing, as much as possible, to detect and treat complications early and to minimize any disability. Current therapies include drugs that focus on the four main features of the disease: inflammation, vascular injury, autoimmunity and tissue fibrosis. These therapies include NSAIDs or corticosteroids, immunosuppressive therapy (methotrexate, cyclosporine, antithymocyte globulin, mycophenolate mofetil and cyclophosphamide), autologous bone marrow transplantation, drug therapy of vascular disease (vasodilator therapy like the calcium channel blockers, angiotensin converting enzyme inhibitors-ACE inhibitors, bosentan - a new endothelin-1 receptor inhibitor, prostacyclin, nitric oxide, anti-coagulation), anti-fibrotic agents (colchicine, D-penicillamine). Of course, for every complication of systemic sclerosis the specific therapy should be used (13).

There has been substantial progress in recent years in the management of some complications developed by scleroderma patients, which has led to increased disease survival and quality of life. This includes better control of complications in specific organs (such as interstitial lung disease, pulmonary arterial hypertension, renal crisis, and Raynaud's phenomenon) as well as standardized follow-up and earlier detection of potential complications (14). This is where the present case report is emphasizing on the importance of dealing with major disease crisis first but focus after on the best management of complications and always consider the therapeutic goal of offering the patient the best quality of life and care that is available, in any circumstances (even in the infectious pandemic context).

The favorable COVID-19 evolution in a patient with multiple organ impairments may be connected with recent findings that in non-severely immunosuppressed individuals (as our patient was under specific therapy for systemic sclerosis) it is less likely to develop moderate or severe ARDS caused by SARS-CoV-2 infection. Multiple studies have now shown that severe COVID-19 is caused by the development of a hyper-inflammatory state, characterized by dysregulation of the immune system and cytokine storm (15). 


\section{CONCLUSIONS}

The patient presented in this case report has a severe form of systemic sclerosis with multiple organ manifestations early after diagnosis, with an evolution that resulted in serious damage of the cardiac and renal function. This type of patient has poorer prognosis because of fast progression of skin and organ involvement including cardiovascular system, lungs, kidneys, gastrointestinal tract and even central and peripheral nervous system. In diffuse systemic sclerosis there is a shorter time period between the onset of Raynaud's phenomenon and skin symptoms. As a result of the wide range of systemic complications, there has been reported that the overall 10 -year sur-

Conflict of interest: none declared

Financial support: none declared

\section{REFERENCES}

1. Careta MF, Romiti R. Localized scleroderma: clinical spectrum and therapeutic update. An Bras Dermatol. 2015 Jan-Feb;90(1):62-73.

2. Denton CP, Khanna D. Systemic sclerosis. Lancet. 2017 Oct 07:390(10103):1685-1699.

3. Arnett FC, Cho M, Chatterjee S, Aguilar MB, Reveille JD, Mayes $\mathrm{MD}$. Familial occurrence frequencies and relative risks for systemic sclerosis (scleroderma) in three United States cohorts. Arthritis Rheum. 2001 Jun;44(6):1359-62.

4. Boin F, Rosen A. Autoimmunity in systemic sclerosis: current concepts. Curr Rheumatol Rep. 2007 May;9(2):165-72.

5. Dumoitier N, Lofek S, Mouthon L. Pathophysiology of systemic sclerosis: state of the art in 2014. Presse Med. 2014 Oct;43(10 Pt 2):e267-78

6. Sierra-Sepúlveda A, Esquinca-González A, Benavides-Suárez SA, Sordo-Lima DE, Caballero-Islas AE, Cabral-Castañeda AR, Rodríguez-Reyna TS. Systemic Sclerosis Pathogenesis and Emerging Therapies, beyond the Fibroblast. Biomed Res Int. 2019 Jan 23;2019:4569826

7. Mukerjee D, St George D, Coleiro B, Knight C, Denton CP, Davar J, Black CM, Coghlan JG. Prevalence and outcome in systemic sclerosis associated pulmonary arterial hypertension: application of a registry approach. Ann Rheum Dis. 2003 Nov;62(11):1088-93.

8. Elhai M, Meune C, Boubaya M, Avouac J, Hachulla E, BalbirGurman A, et AL. Mapping and predicting mortality from systemic sclerosis. Ann Rheum Dis. 2017;76:1897-1905. vival rate ranges from 65 to $82 \%$. The most life-threatening complications are those affecting the heart, kidneys and lungs. Myocardial fibrosis, coronary heart disease, right and left heart failure, arrhythmias, myositis, valvular and pericardial injuries are manifestations of primary heart involvement.

The complexity of the treatment results from the need to inhibit the autoimmune process, inflammation and organ specific management. Although early diagnosis and new therapeutic options significantly improve the prognosis, systemic sclerosis is still characterized by a severe course and high risk of early death.

9. Pokeerbux MR, Giovannelli J, Dauchet L, Mouthon L, Agard C, Lega JC, Allanore Y, Jego P, Bienvenu B, Berthier S, Mekinian A, Hachulla $E$, Launay D. Survival and prognosis factors in systemic sclerosis: data of a French multicenter cohort, systematic review, and metaanalysis of the literature. Arthritis Res Ther. 2019 Apr 3;21(1):86.

10. Bhattacharyya $\mathrm{S}$, Varga J. Emerging roles of innate immune signaling and toll-like receptors in fibrosis and systemic sclerosis. Curr Rheumatol Rep. 2015 Jan;17(1):474.

11. Odonwodo A, Badri T, Hariz A. Scleroderma. [Updated 2020 Aug 8]. In: StatPearls [Internet]. Treasure Island (FL): StatPearls Publishing; 2020 Jan.

12. Azathioprinum - Summary of Product Characteristics updated June 2017. Available at: https://www.anm.ro/_/RCP/ RCP 10042 15.06.17.pdf.

13. Kowal-Bielecka O, Fransen J, Avouac J, Becker M, Kulak A, Allanore Y, Distler O, Clements P, et al.; EUSTAR Coauthors. Update of EULAR recommendations for the treatment of systemic sclerosis. Ann Rheum Dis. 2017 Aug;76(8):1327-1339.

14. Denton CP. Systemic sclerosis: from pathogenesis to targeted therapy. Clin Exp Rheumatol. 2015 Jul-Aug;33(4 Suppl 92):S3-7.

15. Monreal E, Sainz de la Maza S, Fernández-Velasco JI, NateraVillalba E, Rita CG, et al.; COVID-HRC group. The Impact of Immunosuppression and Autoimmune Disease on Severe Outcomes in Patients Hospitalized with COVID-19. J Clin Immunol. 2021 Feb;41(2):315-323. 